\title{
Larvas phyllosoma (Decapoda, Palinuridae y Scyllaridae) de las islas oceánicas chilenas
}

\author{
Jorge Rivera $^{1} \&$ Armando Mujica ${ }^{2}$ \\ ${ }^{1}$ Museo del Mar, Departamento de Ciencias del Mar, Universidad Arturo Prat \\ Casilla 121, Iquique, Chile \\ ${ }^{2}$ Facultad de Ciencias del Mar, Universidad Católica del Norte. Casilla 117, Coquimbo, Chile
}

RESUMEN. Se analizan las larvas phyllosoma de los géneros Jasus, Scyllarus, Projasus (phyllosoma X) y un género indeterminado de la familia Scyllaridae, colectadas durante los cruceros Cimar 5 y 6, Islas Oceánicas chilenas. Se entrega información de la distribución y el estado actual del conocimiento de estas larvas en aguas chilenas. Se incluye una clave de identificación para larvas phyllosoma de los géneros reportados de las áreas de estudio.

Palabras clave: larvas phyllosoma, distribución, islas oceánicas, Chile.

\section{Phyllosoma larvae (Decapoda, Palinuridae and Scyllaridae) from Chilean oceanic islands}

\begin{abstract}
Phyllosoma larvae collected during the Cimar 5 and 6 cruises near Chilean oceanic islands are discussed. They include representatives of the genera Jasus, Scyllarus, Projasus (phyllosoma X) and an indeterminate genus of Scyllaridae. Information is presented on their distribution and the present state of the knowledge of this larval group in Chilean waters. A key for the identification of genera of the phyllosoma larvae reported from the study area is included.
\end{abstract}

Key words: phyllosoma larvae, distribution, oceanic islands, Chile.

\section{INTRODUCCIÓN}

Según lo señalado en la literatura, en las aguas de las islas oceánicas chilenas (Pacífico suroriental) existen ocho especies de langostas marinas, de las cuales Jasus frontalis H. Milne Edwards, Panulirus pascuensis Reed, y Projasus bahamondei George, pertenecen a la familia Palinuridae, mientras que Scyllarus delfíni Bouvier, Ibacus peroni Leach, Parribacus perlatus Holthuis, Scyllarides roggeveeni Holthuis y Arctides regalis Holthuis, son componentes de la familia Scyllaridae (Báez, 1973; Retamal, 1981, 1994, 2000a, 2000b). De éstas, solo la presencia de Ibacus peroni requiere ser confirmada en aguas chilenas (G. Guzmán, com. pers.), encontrándose el resto de las especies distribuidas

Autor corresponsal: Jorge Rivera (jorge.rivera@unap.cl) principalmente en las islas oceánicas chilenas y sus alrededores (Retamal, 1981, 1994, 2000a, 2000b).

La etapa larvaria de estas especies es relativamente larga, con 7 a 9 estadios en Scyllaridae y entre 11 a 17 estadios en Palinuridae, lo que significa un periodo entre 6 y 11 meses de vida planctónica respectivamente (Atkinson \& Boustead, 1982; Ito \& Lucas, 1990; Kittaka et al., 1997).

Los antecedentes sobre morfología y distribución geográfica de las larvas phyllosoma de estos decápodos para las aguas del Pacífico sudoriental son escasos (Wehrtmann \& Báez, 1997), destacando en este aspecto los trabajos de Báez (1973) sobre los phyllosoma de Jasus frontalis, phyllosoma X y Scyllarus delfini. Báez (1979), describe el puerulus (estadio postlarval) de Jasus frontalis y posterior- 
mente, Báez \& Ruiz (2000) describen el puerulus de Projasus bahamondei. Estos estudios, además de otros, han proporcionado información del ciclo de vida, distribución, ciclos reproductivos y aspectos relacionados con el stock-reclutamiento de estas especies (Sastry, 1982; Palma, 1994; Anger, 2001).

El presente trabajo entrega información sobre la distribución de larvas phyllosoma colectadas en torno a las islas oceánicas de Chile (Cruceros Cimar 5 y 6), la relación de éstas con los principales sistemas de corrientes de aguas oceánicas y una clave para la identificación de larvas phyllosoma de los ocho géneros reportados en la zona de estudio.

\section{MATERIALES Y MÉTODOS}

Se recolectaron muestras planctónicas y micronectónicas durante el desarrollo de los cruceros oceanográficos Cimar 5 y 6 Islas Oceánicas, a bordo del buque oceanográfico de la Armada de Chile "Vidal Gormaz". Las muestras se obtuvieron en estaciones oceanográficas preestablecidas en dos transectas oceánicas. La primera, efectuada durante el crucero Cimar 5, unió Caldera $\left(26^{\circ} 59^{\prime} \mathrm{S}\right.$, $71^{\circ} 46^{\prime} \mathrm{W}$ ) con la isla de Pascua, y de allí a la isla Salas y Gómez (265'소 $\left.107^{\circ} 35^{\prime} \mathrm{W}\right)$. La segunda, efectuada durante el crucero Cimar 6, unió Valparaíso $\left(33^{\circ} 00^{\prime} \mathrm{S}, 71^{\circ} 35^{\prime} \mathrm{W}\right)$ con el archipiélago de Juan Fernández ( $\left.33^{\circ} 37^{\prime} S ; 80^{\circ} 00^{\prime} \mathrm{W}\right)$ y de éste a las islas Desventuradas, San Félix y San Ambrosio $\left(26^{\circ} 20^{\prime} \mathrm{S}, 80^{\circ} 00^{\prime} \mathrm{W}\right)$. Todas las muestras se recolectaron durante la temporada de primavera (septiembre-octubre de 1999 y noviembre de 2000) (Figs. 1 y 2).

El material zooplanctónico se capturó mediante arrastres oblicuos realizados entre $200 \mathrm{~m}$ de profundidad y la superficie, utilizando redes Bongo de $60 \mathrm{~cm}$ de diámetro y $300 \mu \mathrm{m}$ de abertura de malla, provistas de medidores de flujos. Además, se efectuaron arrastres entre la superficie y 500-700 m de profundidad, con redes de micronecton IKMT (Isaac-Kid Midwater Trawl) de $9 \mathrm{~m}^{2}$ de abertura de boca, túnel de $10 \mathrm{~m}$ de largo, con malla de $10 \mathrm{~mm}$ y copo de $1000 \mu \mathrm{m}$.

En el crucero Cimar 5, se colectaron muestras de zooplancton en 92 estaciones con redes Bongo, además de 25 muestras obtenidas con red IKMT. En el crucero Cimar 6, en 55 estaciones se obtuvieron muestras con redes Bongo y en 25 de ellas con red IKMT. El número y la hora de captura de larvas phyllosoma en las estaciones de ambos cruceros se indican en las Tablas 1 y 2 , respectivamente.

Las muestras se preservaron en solución de formalina (5\%) neutralizada con bórax. En todas ellas se separaron las larvas Palinuridae y Scyllaridae, las que fueron teñidas con azul de metileno para facilitar su identificación a nivel de especie. La longitud total (LT) se determinó entre el margen anterior del rostro y el borde posterior del telson, mediante el uso de microscopio con ocular graduado.

Los géneros de los phyllosoma de ambas familias se identificaron de acuerdo a los trabajos de Gurney (1936), Johnson (1971), Báez (1973, 1983), Ito \& Lucas (1990), Dupré \& Guisado (1996), Kittaka et al. (1997) y Coutures (2001).

\section{RESULTADOS}

Del total de 197 muestras recolectadas en ambos cruceros, sólo en 28 se obtuvieron larvas phyllosoma, lo cual representa el $14 \%$ de los lances efectuados. La mayoría de las larvas se capturaron durante la noche y se encontraron en estado de phyllosoma, correspondiendo los estadios más desarrollados a las capturas efectuadas con red IKMT. Del total de larvas phyllosoma recolectadas en ambos cruceros (95), sólo cuatro ejemplares (4\%) se capturaron en el crucero Cimar 5 (Fig. 1), los restantes se obtuvieron en el crucero Cimar 6 (96\%) (Fig. 2).

Entre éstas se identificaron cuatro morfos, correspondientes a igual número de especies. De Palinuridae se identificaron larvas de Jasus frontalis (61 phyllosoma) y un ejemplar del género Projasus (phyllosoma X). De Scyllaridae, 31 larvas de Scyllarus delfini, y 2 ejemplares indeterminados, probablemente del género Scyllarides, que por ser phyllosoma de estadio I, con caracteres morfológicos poco definidos, impidió su asignación específica.

Los phyllosoma de Jasus frontalis se capturaron solo en el crucero Cimar 6, principalmente con redes Bongo, aunque los ejemplares de mayor desarrollo y talla se encontraron en las muestras obtenidas con red IKMT.

Los phyllosoma de Scyllarus delfini (31 larvas) fueron las únicas presentes en ambos cruceros y se capturaron principalmente con redes Bongo durante el crucero Cimar 6, encontrándose bien representadas alrededor del archipiélago de Juan Fernández 

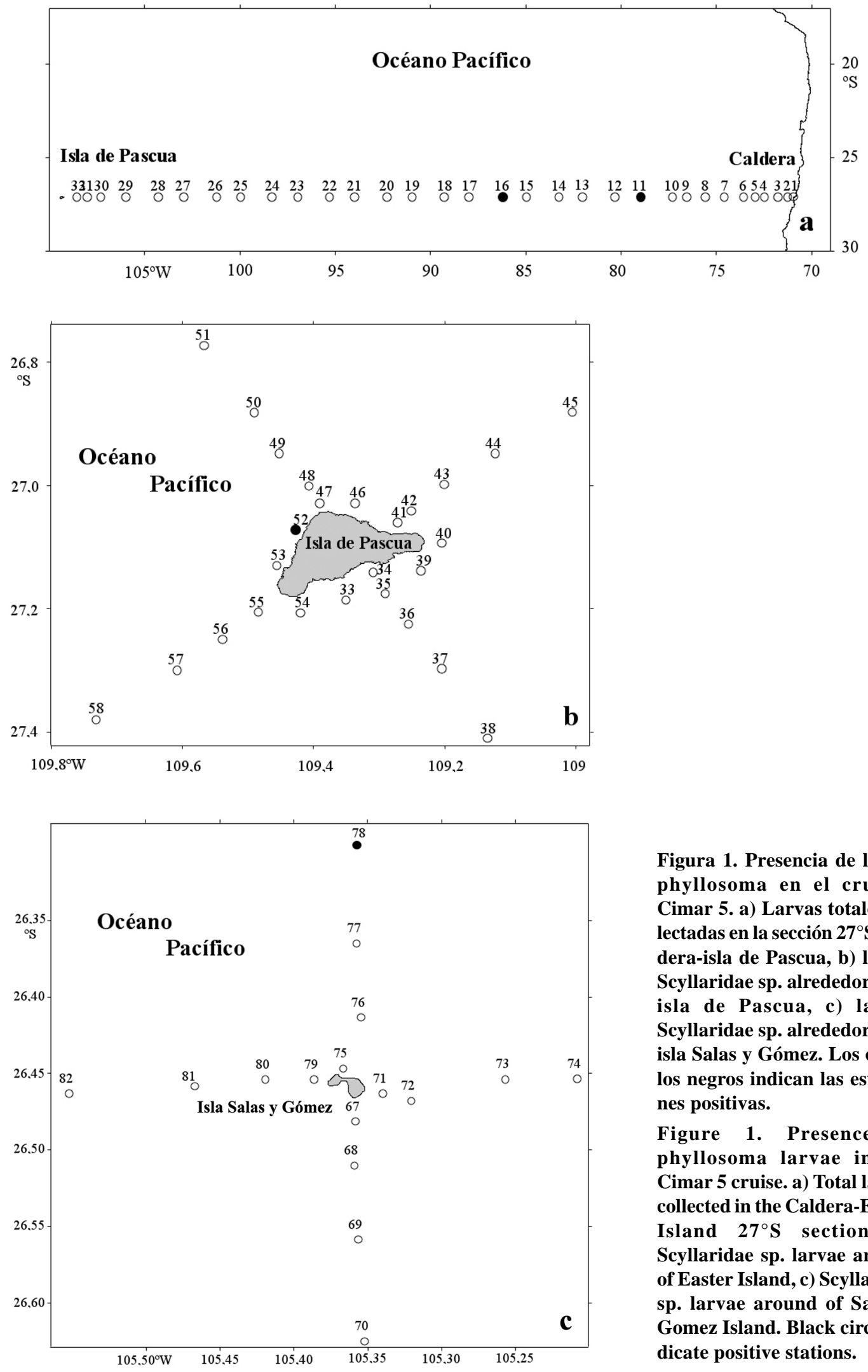

Figura 1. Presencia de larvas phyllosoma en el crucero Cimar 5. a) Larvas totales colectadas en la sección $27^{\circ} \mathrm{S}$ Caldera-isla de Pascua, b) larvas Scyllaridae sp. alrededor de la isla de Pascua, c) larvas Scyllaridae sp. alrededor de la isla Salas y Gómez. Los círculos negros indican las estaciones positivas.

Figure 1. Presence of phyllosoma larvae in the Cimar 5 cruise. a) Total larvae collected in the Caldera-Easter Island $27^{\circ} \mathrm{S}$ section, b) Scyllaridae sp. larvae around of Easter Island, c) Scyllaridae sp. larvae around of Salas y Gomez Island. Black circle indicate positive stations. 
Tabla 1. Estaciones del crucero Cimar 5, con presencia de larvas phyllosoma.

Table 1. Stations of the Cimar 5 cruise, with occurrence of phyllosoma larvae.

\begin{tabular}{|c|c|c|c|c|c|c|c|c|c|}
\hline \multirow[t]{2}{*}{ Estación } & \multicolumn{2}{|c|}{ Scyllarus delfini } & \multicolumn{2}{|c|}{ Projasus sp. } & \multicolumn{2}{|c|}{ Scyllaridae sp. } & \multirow[t]{2}{*}{ Total } & \multirow{2}{*}{$\begin{array}{c}\text { Hora } \\
\text { local } \\
\text { (GMT } \mathbf{- 0 4 : 0 0 )} \text { ) }\end{array}$} & \multirow[t]{2}{*}{ Localidad } \\
\hline & Bongo & IKMT & Bongo & IKMT & Bongo & IKMT & & & \\
\hline 11 & 1 & $\mathrm{x}$ & $\mathrm{x}$ & $\mathrm{x}$ & $\mathrm{x}$ & $\mathrm{x}$ & 1 & 05:00 05:10 & Sección $27^{\circ} \mathrm{S}$ \\
\hline 16 & $\mathrm{x}$ & $\mathrm{x}$ & $\mathrm{x}$ & 1 & $\mathrm{x}$ & $\mathrm{x}$ & 1 & $23: 54 \quad 00: 13$ & Sección $27^{\circ} \mathrm{S}$ \\
\hline 52 & $\mathrm{x}$ & $\mathrm{x}$ & $\mathrm{x}$ & $\mathrm{x}$ & 1 & $\mathrm{x}$ & 1 & 03:41 $03: 57$ & Isla de Pascua \\
\hline 78 & $\mathrm{x}$ & $\mathrm{x}$ & $\mathrm{x}$ & $\mathrm{x}$ & $\mathrm{x}$ & 1 & 1 & 21:03 21:18 & I. Salas y Gómez \\
\hline Total & 1 & 0 & 0 & 1 & 1 & 1 & 4 & & \\
\hline
\end{tabular}

Tabla 2. Estaciones del crucero Cimar 6, con presencia de larvas phyllosoma.

Table 2. Stations of the Cimar 6 cruise, with occurrence of phyllosoma larvae.

\begin{tabular}{|c|c|c|c|c|c|c|c|}
\hline \multirow[t]{2}{*}{ Estación } & \multicolumn{2}{|c|}{ Jasus frontalis } & \multicolumn{2}{|c|}{ Scyllarus delfini } & \multirow[t]{2}{*}{ Total } & \multirow{2}{*}{$\begin{array}{c}\text { Hora local } \\
\text { (GMT 04:00) }\end{array}$} & \multirow[t]{2}{*}{ Localidad } \\
\hline & Bongo & IKMT & Bongo & IKMT & & & \\
\hline 5 & $\mathrm{x}$ & 1 & $\mathrm{x}$ & $\mathrm{x}$ & 1 & 05:10 08:00 & Sección $33^{\circ} \mathrm{S}$ \\
\hline 26 & 8 & $\mathrm{x}$ & 2 & $\mathrm{x}$ & 10 & $22: 30 \quad 23: 00$ & I. Robinson Crusoe \\
\hline 30 & 1 & $\mathrm{x}$ & $\mathrm{x}$ & $\mathrm{x}$ & 1 & 01:50 $04: 15$ & I. Robinson Crusoe \\
\hline 27 & 2 & $\mathrm{x}$ & $\mathrm{x}$ & $\mathrm{x}$ & 2 & 07:10 07:50 & I. Robinson Crusoe \\
\hline 35 & 2 & $\mathrm{x}$ & 3 & $\mathrm{x}$ & 5 & 08:50 09:30 & I. Robinson Crusoe \\
\hline 33 & $\mathrm{x}$ & $\mathrm{x}$ & 1 & $\mathrm{x}$ & 1 & 09:55 10:30 & I. Robinson Crusoe \\
\hline 32 & 1 & $\mathrm{x}$ & 1 & $\mathrm{x}$ & 2 & $15: 50 \quad 16: 30$ & I. Robinson Crusoe \\
\hline 40 & 7 & $\mathrm{x}$ & 1 & $\mathrm{x}$ & 8 & $18: 20 \quad 18: 45$ & I. Robinson Crusoe \\
\hline 39 & 3 & $\mathrm{x}$ & 1 & $\mathrm{x}$ & 4 & $19: 15 \quad 19: 45$ & I. Robinson Crusoe \\
\hline 38 & 2 & $\mathrm{x}$ & 1 & $\mathrm{x}$ & 3 & $20: 10 \quad 20: 40$ & I. Robinson Crusoe \\
\hline 36 & 4 & $\mathrm{x}$ & 1 & $\mathrm{x}$ & 5 & 21:00 22:00 & I. Robinson Crusoe \\
\hline 13 & 1 & 1 & $\mathrm{x}$ & 1 & 3 & 06:00 08:25 & Sección $33^{\circ} \mathrm{S}$ \\
\hline 19 & 4 & $\mathrm{x}$ & 1 & $\mathrm{x}$ & 5 & $11: 40 \quad 13: 45$ & I. Alejandro Selkirk \\
\hline 18 & $\mathrm{x}$ & $\mathrm{x}$ & 2 & $\mathrm{x}$ & 2 & 16:05 16:30 & I. Alejandro Selkirk \\
\hline 20 & 2 & $\mathrm{x}$ & $\mathrm{x}$ & $\mathrm{x}$ & 2 & 18:20 19:10 & I. Alejandro Selkirk \\
\hline 17 & 10 & $\mathrm{x}$ & 1 & $\mathrm{x}$ & 11 & 22:10 01:00 & I. Alejandro Selkirk \\
\hline 56 & 7 & $\mathrm{x}$ & 3 & $\mathrm{x}$ & 10 & $16: 35 \quad 16: 55$ & Is. Desventuradas \\
\hline 55 & 1 & $\mathrm{x}$ & 4 & $\mathrm{x}$ & 5 & $17: 15 \quad 17: 38$ & Is. Desventuradas \\
\hline 53 & $\mathrm{x}$ & $\mathrm{x}$ & 2 & $\mathrm{x}$ & 2 & $18: 15 \quad 18: 40$ & Is. Desventuradas \\
\hline 54 & $\mathrm{x}$ & $\mathrm{x}$ & 1 & $\mathrm{x}$ & 1 & 19:27 21:05 & Is. Desventuradas \\
\hline 52 & $\mathrm{x}$ & $\mathrm{x}$ & 1 & $\mathrm{x}$ & 1 & $22: 18 \quad 01: 40$ & Is. Desventuradas \\
\hline 58 & 2 & $\mathrm{x}$ & $\mathrm{x}$ & $\mathrm{x}$ & 2 & 02:49 03:41 & Is. Desventuradas \\
\hline 59 & $\mathrm{x}$ & $\mathrm{x}$ & 1 & $\mathrm{x}$ & 1 & $10: 45 \quad 11: 22$ & Is. Desventuradas \\
\hline 51 & 2 & $\mathrm{x}$ & 2 & $\mathrm{x}$ & 4 & $12: 00 \quad 14: 10$ & Is. Desventuradas \\
\hline Total & 59 & 2 & 29 & 1 & 91 & & \\
\hline
\end{tabular}




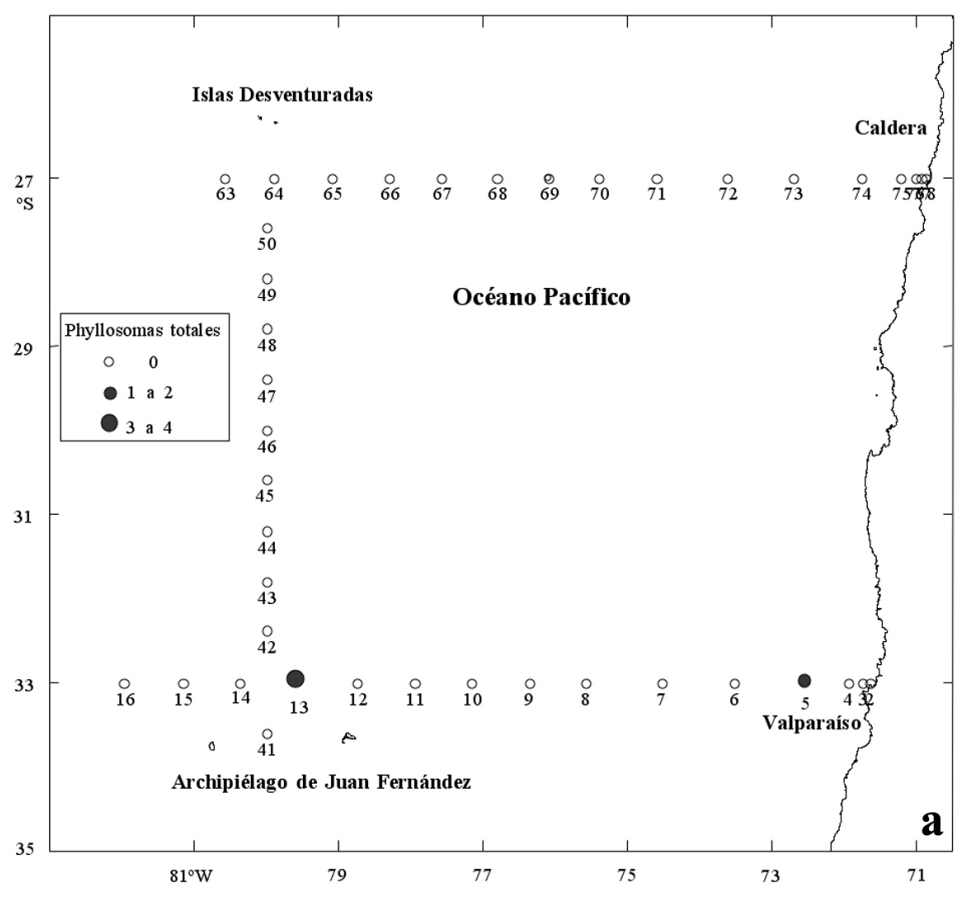

Figura 2. Distribución de larvas phyllosoma en el crucero Cimar 6. a) En la sección $27^{\circ} \mathrm{S}$ (Caldera-islas Desventuradas), sección $33^{\circ} \mathrm{S}$ (Valparaíso-archipiélago de Juan Fernández) y sección $80^{\circ} \mathrm{W}$ (archipiélago de Juan Fernández-islas Desventuradas), b) alrededor del archipiélago de Juan Fernández, c) alrededor de las islas Desventuradas.

Figure 2. Distribution of phyllosoma larvae in the Cimar 6 cruise. a) In the $27^{\circ} \mathrm{S}$ section (Caldera-Desventuradas Islands), $33^{\circ} \mathrm{S}$ section (Valparaíso-Juan Fernández archipelago) and $80^{\circ} \mathrm{W}$ section (Juan Fernández archipelago-Desventuradas Islands), b) around of Juan Fernández Archipelago, c) around of Desventuradas Islands.
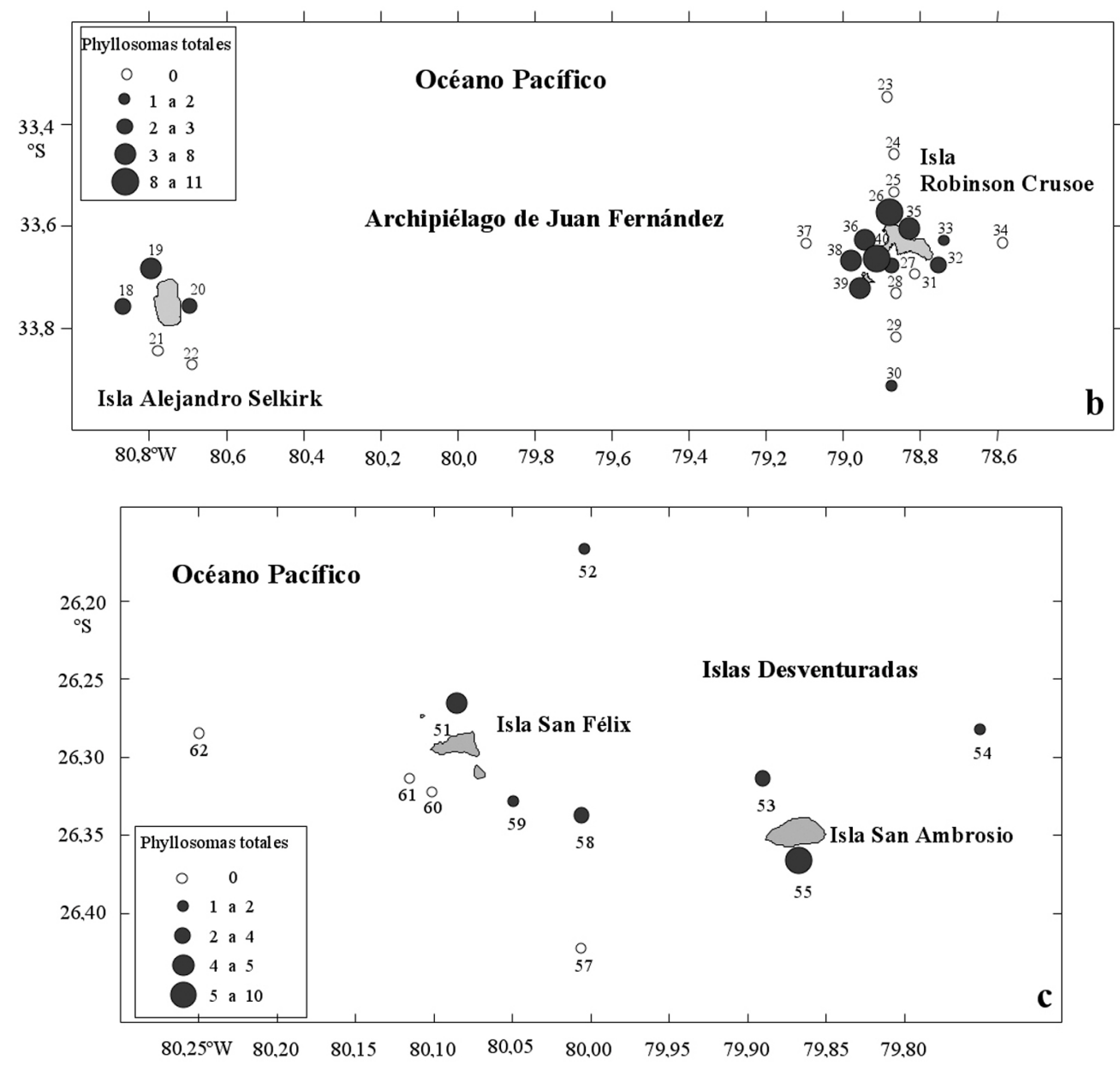
y de las islas Desventuradas (Figs. 2b y 2c). En el crucero Cimar 5 sólo se capturó un ejemplar con redes Bongo, en la estación 11 cercana a las islas Desventuradas (Fig. 1c). Las larvas de esta especie capturadas con redes Bongo se encontraron en estados de desarrollo comprendido entre phyllosoma I y V, inferiores a $8 \mathrm{~mm}$ (LT), mientras que el ejemplar capturado con redes IKMT, correspondió a phyllosoma V, de 8,24 mm (LT).

Las larvas del género Projasus (phyllosoma X) $\mathrm{y}$ las Scyllaridae indeterminadas, se capturaron solo durante el crucero Cimar 5. El primero de estos morfotipos se obtuvo con red IKMT, en una estación ubicada en la mitad del transecto entre Caldera e isla de Pascua (Fig. 1a). Esta larva pertenecía a phyllosoma VII u VIII y su talla fue de 11,64 mm de LT.

Las larvas de Scyllaridae indeterminadas se recolectaron en dos estaciones, al oeste de isla de Pascua y al norte de la isla Salas y Gómez (Figs. 1b y 1c), capturadas con redes Bongo e IKMT respectivamente, ambas como phyllosoma I, de tallas 1,20 y 1,23 mm. Los estadios de desarrollo y los rangos de las mediciones de la longitud total de los ejemplares colectados se resumen en la Tabla 3.

\section{Clave de identificación para larvas phyllosoma de los géneros presentes alrededor de las islas oceánicas chilenas}

Esta clave se elaboró considerando los antecedentes del presente trabajo y los de Gurney (1936), Johnson (1971), Báez (1973; 1983), Ritz \& Thomas (1973), Atkinson \& Boustead (1982), Ito \& Lucas (1990), Dupré \& Guisado (1996), Kittaka et al. (1997) y Coutures (2001). (*): tricotomía.

1. Maxilípedos 2 y 3 con exópodos bien desarrollados y setosos.

Maxilípedos 2 y 3 con exópodos rudimentarios o poco desarrollados sin setas 3

2. Cephalon circular; tórax menos ancho que el cephalon; segmento basal de la antena con una espina en el borde distal externo en los estadios avanzados phyllosoma X (Projasus) Cephalon piriforme, angosto en su parte anterior; tórax más ancho o igual que el cephalon; segmento basal de la antena liso sin espina en los estadios avanzados

Panulirus

3. Antenas más o tan largas como las anténulas, segmento basal del pedúnculo antenal nunca más ancho que los demás segmentos, flagelo antenal largo y delgado en los estadios avanzados Jasus Antenas más cortas que las anténulas; segmento

Tabla 3. Referencias y datos de captura de las larvas phyllosoma identificadas en las estaciones de los cruceros Cimar 5 y 6.

Table 3. References and capture data from phyllosoma larvae identified at the stations of the Cimar 5 and 6 cruises.

\begin{tabular}{|c|c|c|c|c|c|c|}
\hline \multirow[t]{2}{*}{ Especie } & \multirow[t]{2}{*}{ Referencias } & \multirow{2}{*}{$\begin{array}{c}\mathbf{N}^{\circ} \\
\text { larvas }\end{array}$} & \multicolumn{2}{|c|}{ Estadio } & \multirow{2}{*}{$\begin{array}{l}\text { Longitud } \\
\text { Total } \\
(\mathbf{m m})\end{array}$} & \multirow[t]{2}{*}{ Crucero } \\
\hline & & & $\begin{array}{c}\text { Red } \\
\text { Bongo }\end{array}$ & $\begin{array}{l}\text { Red } \\
\text { IKMT }\end{array}$ & & \\
\hline $\begin{array}{l}\text { Projasus sp. } \\
\text { (phyllosoma X) }\end{array}$ & $\begin{array}{c}\text { Báez, 1973; } \\
\text { MacWilliam (com. pers.) }\end{array}$ & 1 & - & VII u VIII & 11,64 & Cimar 5 \\
\hline Scyllaridae sp. & $\begin{array}{l}\text { Gurney, 1936; } \\
\text { Ito \& Lucas, 1990, } \\
\text { Coutures, } 2001\end{array}$ & 2 & I & I & $1,20-1,23$ & Cimar 5 \\
\hline Scyllarus delfini & $\begin{array}{c}\text { Báez, 1973; } \\
\text { Ito \& Lucas, } 1990\end{array}$ & 31 & $\mathrm{I}-\mathrm{V}$ & V & $1,18-8,24$ & Cimar 5 y 6 \\
\hline Jasus frontalis & $\begin{array}{c}\text { Báez, 1973; } \\
\text { Dupré \& Guisado 1996; } \\
\text { Kittaka et al., } 1997\end{array}$ & 61 & $\mathrm{I}-\mathrm{V}$ & XII & $1,20-31,31$ & Cimar 6 \\
\hline
\end{tabular}


basal del pedúnculo antenal más ancho que los demás segmentos, flagelo antenal corto y ancho en los estadios avanzados 4

4. Pereiópodos con espinas subexopodales; cefalon más ancho que largo...................................5 Pereiópodos sin espinas subexopodales; cephalon más largo que ancho $6(*)$

5. Cephalon sin hendidura anterior, bordes láteroposteriores del cephalon cubren solo los coxapoditos del primer par de pereiópodos en los estadios avanzados, borde posterior del cephalon proyectado hacia el tórax como una cúspide redondeada; $5^{\circ}$ par de pereiópodos sin exópodo; abdomen amplio en su base y con bordes laterales cóncavos.. Scyllarus

Cephalon hendido anteriormente (forma de manzana), bordes láteroposteriores del cephalon cubren los coxapoditos del segundo y tercer par de pereiópodos en los estadios avanzados, borde posterior recto o hendido levemente; pereriópodo 5 con exópodo setoso, abdomen tan ancho como largo, sin bordes laterales cóncavos. Ibacus

6. Porción anterior del cephalon ancha, borde posterior sobresaliente en los estadios tempranos; tórax más angosto que el cephalon, ligeramente hendido en su borde posterior solo en los últimos estadios; abdomen amplio, con solo la base inserta dentro de la hendidura del tórax ................ Scyllarides Porción anterior del cephalon angosta, borde posterior no sobresale en estadios tempranos; tórax más ancho que el cephalon, profundamente hendido en su borde posterior; abdomen angosto y pequeño, completamente inserto en la hendidura posterior del tórax Parribacus

(*) Cephalon piriforme, rostro redondeado; segmento distal de la anténula alcanza 1/3 del largo total del apéndice en el estadio I ..... Arctides

\section{DISCUSIÓN}

\section{Distribución de larvas phyllosoma}

El escaso número de larvas phyllosoma encontradas en las muestras de ambos cruceros, capturadas tanto con redes Bongo (ambiente epipelágico), como con red IKMT (ambiente mesopelágico), es algo que ha sido destacado con anterioridad para las aguas circundantes al archipiélago de Juan Fernández (Báez, 1973; Palma et al., 1976). Sin embargo, las pequeñas cantidades de phyllosoma capturadas en esta ocasión superan ampliamente a las capturas realizadas por los autores citados.

La baja capturabilidad de estas larvas sería motivada por su gran movilidad dada su morfología y adaptaciones a la vida pelágica, lo que les permitiría evadir las redes que normalmente se usan para su captura. Esto, sumado a la distribución espacial tanto vertical como horizontal, además de la distribución temporal en el plancton, hace que tanto su accesibilidad como disponibilidad sea otro aspecto que interfiere en su capturabilidad.

En este sentido, Rimmer \& Phillips (1979) determinaron que los phyllosoma de Panulirus cygnus alcanzan velocidades de 16,6 y $20 \mathrm{~m} \cdot \mathrm{h}^{-1}$, en los estadios intermedios y avanzados, respectivamente. Dupré \& Guisado (1996), señalan que el phyllosoma I de Jasus frontalis, desarrolla velocidades entre 0,54 y $0,75 \mathrm{~m} \cdot \mathrm{min}^{-1}$. Las velocidades de escape que los zooplancteres ejecutan por breves instantes son difíciles de determinar. Sin embargo, Ohman (1988) indica que entre las estrategias que desarrollan los zooplancteres para evitar la depredación está el lograr aceleraciones en fracciones de segundo que superan las velocidades de arrastre de las redes.

La distribución vertical diaria de las larvas y su relación con la luz disponible en diferentes profundidades y hora del día, es otro de los aspectos que debe ser considerado en la capturabilidad y evasión de estas larvas. Rimmer \& Phillips (1979) han descrito la migración vertical de larvas de Panulirus cygnus, destacando la relación de la luz con la profundidad de distribución de los phyllosoma. Ellos encontraron las mayores densidades de larvas entre 0 y $10 \mathrm{~m}$ durante noches de luna nueva, y un desplazamiento a profundidades entre 50 y $80 \mathrm{~m} \mathrm{du}$ rante las noches de luna llena en el mismo horario (entre las 20 y 04 h). Durante el día las larvas se dispersan a profundidades comprendidas entre 100 y $200 \mathrm{~m}$.

La distribución vertical diaria y la visión de estas larvas sería una estrategia que les permitiría evadir la depredación y por ende la captura con las redes. Se debe señalar que la mayoría de los lances efectuados en estos cruceros se realizaron en horario diurno, mientras que la mayoría de las capturas de phyllosoma se efectuaron en lances nocturnos al atardecer o en la madrugada (Tablas 1 y 2).

Báez (1983) encontró las mayores densidades de phyllosoma de Panulirus gracilis en aguas tropicales de Costa Rica, en lances efectuados durante 
la noche, a diferencia de lo reportado por Yeung \& McGowan (1991), quienes no encontraron diferencias significativas entre capturas diurnas y nocturnas frente a los cayos de Florida en el Atlántico noroccidental. No obstante, estos autores destacan diferencias significativas existentes tanto en la distribución horizontal de los diversos géneros recolectados, como en la distribución batimétrica de los estadios de desarrollo.

Las larvas phyllosoma de los géneros recolectados en esta oportunidad se encontraron en lugares que coinciden con la distribución de los adultos en el área de estudio. Los phyllosoma de Jasus frontalis y Scyllarus delfini se colectaron solo en áreas próximas a la costa de las islas en que han sido señalados los adultos (Retamal, 1981, 1994, 2000; Rozbaczylo \& Castilla, 1987). Esta distribución, asociada a aguas cercanas, tanto a las islas Desventuradas como a las del archipiélago de Juan Fernández, tendría relación con el endemismo de estas especies (Rozbaczylo \& Castilla, 1987).

A diferencia del género Scyllarus, los phyllosomas del género Panulirus se distribuirían en aguas más alejadas de las costa (Yeung \& McGowan, 1991), acercándose a ellas solo en estadios postlarvales o puerulus para su posterior asentamiento. Esta sería una posible explicación de la ausencia de larvas de Panulirus pascuensis encontrada en los trabajos de los cruceros rusos realizados en el área de los montes submarinos de la placa de Nazca, y de Salas y Gómez (Vereschaka, 1990, 1995; Parin et al., 1997), como en los trabajos de Mujica (1993) y de los cruceros Cimar, los cuales prospectaron las aguas circundantes a la isla de Pascua, sin haber encontrado larvas de P. pascuensis, especie considerada endémica de la isla de Pascua y de la isla Pitcairn (Retamal, 1981; Castilla \& Rozbaczylo, 1987; Pollock, 1992).

La distribución de la denominada phyllosoma $\mathrm{X}$ dentro del transecto entre Caldera e isla de Pascua durante el crucero Cimar 5, concuerda con la distribución señalada por Parin et al. (1997), para los adultos de Projasus bahamondei, reportando una gran dominancia numérica para el sector este del cordón de montes submarinos de Salas y Gómez, entre los $25^{\circ} \mathrm{S}$ y $84^{\circ} \mathrm{W}$.

La identificación de la larva denominada phyllosoma $\mathrm{X}$ ha generado controversia en la asignación de género al que pertenecería. Siendo asignada tentativamente como perteneciente a Projasus parkeri (Báez, 1973), puesto que P. bahamondei no había sido descrita aún. Más tarde, Webber \& Booth (1988) descartan que la phyllosoma X corresponda a Projasus bahamondei, indicando que podría pertenecer a otra especie de palinúrido. Al respecto, se puede señalar que las características morfológicas del phyllosoma X, difieren de los morfotipos larvales descritos para Jasus y Panulirus (McWilliam, 1995; Pollock, 1995; Kittata el al., 1997; Dupré \& Guisado, 1996; P. McWilliam com. pers.). Estos antecedentes, sumado a lo aportado por Parin et al. (1997), permite postular que el phyllosoma $\mathrm{X}$ es una larva morfológicamente más próxima al género Projasus que a otros de la familia Palinuridae, el cual se encuentra presente en las áreas de captura de estas larvas.

La presencia de un ejemplar de la familia Scyllaridae durante el crucero Cimar 5 en los alrededores de la isla Salas y Gómez, se ajusta a la distribución de tres especies de esciláridos reportados para la zona: Arctides regalis, Parribacus perlatus y Scyllarides roggeveeni (Retamal, 1981, 2000a, 2000b), siendo más probable que pertenezca a la ultima especie, dado algunos detalles morfológicos que corresponden al género Scyllarides. Sin embargo, por ser estas larvas de un estadio temprano de desarrollo, no se puede asegurar su pertenencia específica.

Respecto de la distribución vertical de las diferentes especies o morfotipos larvales capturados, se puede señalar, en general, que los estadios tempranos fueron capturados entre 0 y $200 \mathrm{~m}$ de profundidad, mientras que los phyllosoma más desarrollados se encontraron en los lances efectuados hasta $700 \mathrm{~m}$ de profundidad. Esto coincide con lo encontrado por Yeung \& McGowan (1991) respecto a la distribución vertical de phyllosoma en la costa de Florida, donde las mayores concentraciones de estadios tempranos se encontraron en los primeros $50 \mathrm{~m}$, aumentando la incidencia de estadios avanzados al aumentar la profundidad hasta $175 \mathrm{~m}$.

Esto puede tener diferentes interpretaciones, puesto que la capacidad de escape de los ejemplares más grandes o desarrollados haría menos probable su captura en profundidades donde puede ser visible la red a mayor distancia, lo cual disminuiría con la profundidad, así como también con las horas de oscuridad. Por último es necesario considerar la diferente velocidad y tamaño de boca de las redes empleadas en la captura de estas larvas y en especial las usadas en esta oportunidad. 


\section{Consideraciones oceanográficas en la dispersión larval}

La dispersión de los estadios larvales y el reclutamiento de palinúridos y esciláridos en ambientes pelágicos tiene relación directa con la duración del desarrollo larvario de las distintas especies, con los sistemas de corrientes y los mecanismos oceanográficos de cada océano, los que permiten la formación de parches de distribución en diferentes escalas de tiempo y espacio (Saisho, 1966; Johnson, 1971; Sastry, 1981).

Las condiciones oceanográficas presentes en las aguas del Pacífico sudoriental han sido relativamente bien estudiadas por diferentes autores, los cuales han caracterizado las masas de aguas y flujos de corrientes presentes entre las islas oceánicas y el continente (Silva \& Sievers, 1974; Sievers \& Silva, 1975; Silva \& Konow, 1975; Silva, 1992; Moraga \& Olivares, 1996). Frente al continente, entre la costa y los $82^{\circ} \mathrm{W}$, superficialmente $(0-100 \mathrm{~m}$ de profundidad) se encuentran aguas subantárticas. Al oeste de los $82^{\circ} \mathrm{W}$, entre los $0-300 \mathrm{~m}$ de profundidad se encuentran aguas subtropicales, las cuales alcanzan las regiones insulares que se encuentran en la zona central del giro anticiclónico del Pacífico sur (R. Fuenzalida, com. pers.).

Las islas Desventuradas y el archipiélago de Juan Fernández, ubicadas entre los $78^{\circ} \mathrm{W}$ y $80^{\circ} \mathrm{W}$, están influenciadas por aguas de la contracorriente subsuperficial Perú-Chile. Al respecto se ha descrito una relativa estacionalidad de las aguas que bañan las islas, que sería inversa entre las islas Desventuradas y archipiélago de Juan Fernández. Las primeras estarían afectadas la mayor parte del año por aguas ecuatoriales, provenientes del norte, excepto en invierno, mientras que en Juan Fernández, la mayor parte del año, las islas son bañadas por las aguas subantárticas, proceso que se invertiría en verano, en que las aguas de origen subtropical se desplazan más al sur alcanzando el archipiélago (Bahamonde, 1987). No obstante, los sistemas de corrientes que se presentan entre la zona norte y centro de Chile, exhiben un sistema de flujos y contraflujos en direcciones norte y sur respectivamente, los cuales se relacionan con las fronteras que separan las aguas frías de las cálidas que se desplazan en sentido opuesto (R. Fuenzalida, com. pers.) (Fig. 3).

En este sentido, las características oceanográficas de esta área serían determinantes en la dispersión y retención de las larvas phyllosoma de Jasus,
Scyllarus y Projasus (phyllosoma X), principalmente en lo que respecta al rango de distribución de las especies de estos géneros entre las islas Desventuradas y el archipiélago de Juan Fernández. Siendo en especial, el sistema de flujos y contraflujos descrito para esta zona una posible limitante de la dispersión hacia aguas más oceánicas, reteniendo estas larvas en aguas de influencia subtropical de la contracorriente subsuperficial Perú-Chile.

Otro aspecto que es determinante en la dispersión de las especies de los géneros antes mencionados es la topografía de fondo, que generaría las condiciones propicias para el asentamiento de las larvas que completan su desarrollo, manteniendo así el proceso de reclutamiento dentro de los cordones de montes submarinos que conforman las islas Desventuradas y el archipiélago de Juan Fernández, actuando éstos como barreras norte y sur, respectivamente.

Por otra parte, las condiciones oceanográficas de isla de Pascua, y de Salas y Gómez, ubicadas al oeste de los $80^{\circ} \mathrm{W}$, han sido descritas en estudios de corta duración (Moraga \& Olivares, 1996; R. Fuenzalida, com. pers.). Donde la circulación asociada a estas islas corresponde a la del giro anticiclónico del Pacífico sur (Parson et al., 1984; Mann \& Lazier, 1991). Este patrón de circulación de las aguas subtropicales, dentro del giro anticiclónico que influencia a isla de Pascua estaría siendo limitado al este por el sistema de flujos y contraflujos antes mencionado, que a su vez, formarían la frontera que separa las aguas frías continentales, de las aguas cálidas oceánicas (R. Fuenzalida, com. pers.). Estas características oceanográficas, además del aislamiento de la isla de Pascua dentro del Pacífico sudoriental, serían las principales razones de su endemismo e impedimento para la dispersión de las larvas phyllosoma de Panulirus pascuensis, Parribacus perlatus, Scyllarides roggeveeni y Arctides regalis, de las cuales las tres primeras son endémicas para la isla (Castilla \& Rozbaczylo, 1987; Pollock, 1992; George, 1997). Estas no alcanzarían las aguas de las islas Desventuradas y del archipiélago de Juan Fernández, debido al sistema de flujos y contraflujos cuya detección en los cruceros Cimar 5 y 6 fue establecido a los $82^{\circ} \mathrm{W}$ y $100^{\circ} \mathrm{W}$, respectivamente (R. Fuenzalida, com. pers.).

Por otra parte, si la isla de Pascua se encuentra en el centro del giro anticiclónico del Pacífico sur, la variación de intensidad y desplazamiento geográfico temporal explicaría el proceso de retención de 


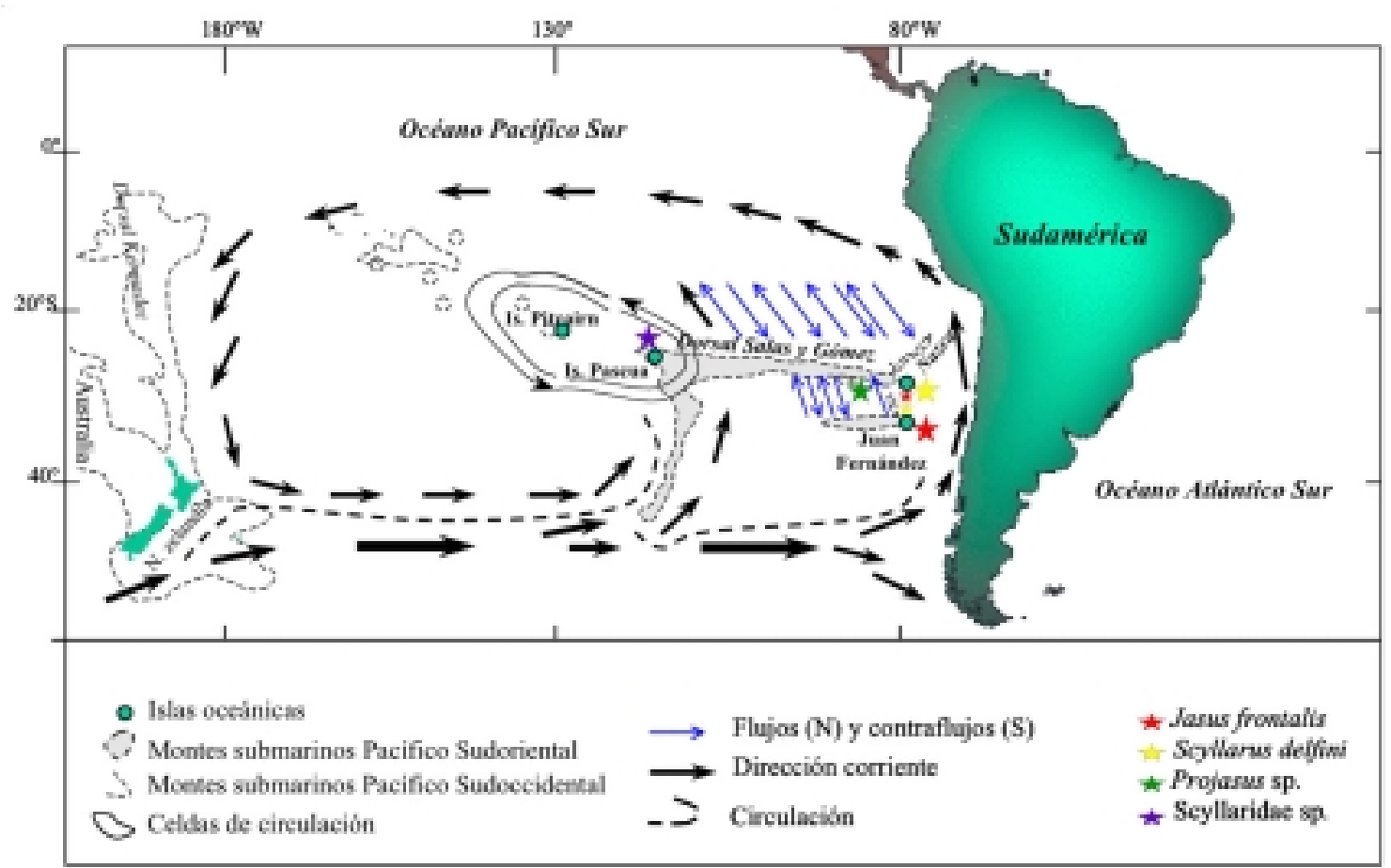

Figura 3. Circulación, corrientes, montes submarinos y distribución de larvas phyllosoma en islas oceánicas de Chile (modificado de Pollock, 1992).

Figure 3. Circulation, currents, seamount chains and distribution of phyllosoma larvae from Chilean oceanic islands (redraw after Pollock, 1992).

la mayor parte del meroplancton en las costas insulares. Mientras que la dispersión de las especies con larvas de desarrollo prolongado se verían favorecidas por el sistema de celdas de circulación formados por las cadenas de montes submarinos del Pacífico sudeste, como son la dorsal Salas y Gómez, a la cual pertenece isla de Pascua y los montes submarinos alrededor de la isla Pitcairn (Pollock, 1992).

Estos sistemas de celdas de circulación, según lo señalado por Pollock (1990, 1992) y Cobb (1997), serían favorables para la dispersión larval y la posterior metamorfosis de los puerulus y su asentamiento en el fondo, que sería gatillado por factores ambientales específicos de hábitat nativos, como serían las costas de las islas de Pascua y Pitcairn, en especial para el caso de las larvas de Panulirus pascuensis endémica de estas islas.

El desarrollo extendido de las larvas de palinúridos y esciláridos, conjuntamente con los patrones de circulación oceánica, son factores críticos para el reclutamiento de las poblaciones de langostas marinas. Los primeros phyllosoma serían transportados mar afuera por las corrientes superficiales, mientras que los estadios intermedios y avanzados estarían regidos por la circulación de las aguas bajo las capas superficiales, que los conducirían a áreas cercanas a las islas y montes submarinos para su reclutamiento (Phillip et al., 1978).

Estos sistemas de circulación, que determinarían la dispersión larval y el reclutamiento dentro en las aguas de las islas oceánicas chilenas, concuerdan con los mecanismos descritos por Saisho (1966), Johnson (1971) y Richards \& Goulet (1976), para la dispersión y reclutamiento de larvas phyllosomas en el Pacífico y Atlántico. Una de las posibilidades es la de un sistema abierto de corta trayectoria que incluye las corrientes y contracorrientes paralelas a la costa (Menzies \& Kerrigan, 1978; Cobb, 1997), manteniendo el transporte y el asentamiento larval dentro de las localidades del rango de distribución de las especies, tal como estaría ocurriendo en torno a las islas Desventuradas y el archipiélago de Juan Fernández. La otra posibilidad implica un sistema cerrado de circulación de larga trayectoria, 
donde las larvas son transportadas dentro de celdas de circulación, para luego retornar en el momento de completar su desarrollo y reclutar a la población adulta (Pollock, 1990, 1992; Cobb, 1997), situación que sería la imperante en el centro del giro anticiclónico del Pacífico sur, donde están las islas de Pascua, y Salas y Gómez.

En general, se puede señalar que los antecedentes relacionados con la distribución espacial y temporal de las larvas phyllosoma en aguas oceánicas chilenas son aún muy escasos. Es necesario efectuar investigaciones más profundas mediante metodologías adecuadas de muestreo, como son el uso de redes con apertura y cierre múltiple, para obtener mayor información tanto de la distribución vertical como horizontal de las larvas, así como de la determinación de sus áreas de desove, las que entregarían mejores antecedentes sobre la dinámica meroplanctónica de los crustáceos decápodos de las islas oceánicas de Chile.

\section{AGRADECIMIENTOS}

Los autores expresan sus más sinceros agradecimientos al Comité Oceanográfico Nacional por el desarrollo de los cruceros de investigación científica marina Cimar 5 y 6 Islas Oceánicas, los cuales han permitido la elaboración de trabajos que contribuyen al conocimiento de los ecosistemas marinos oceánicos. De igual manera a los profesores Walter Sielfeld y Guillermo Guzmán, de la Universidad Arturo Prat, por el apoyo y generosidad hacia las nuevas generaciones de científicos y su destacada participación en estos cruceros. También, agradecemos a la Dra. Paulette McWilliam por sus valiosos comentarios. Un especial reconocimiento a los ayudantes del laboratorio de zooplancton de la Universidad Católica del Norte, Coquimbo, Constanza, Anahí, Humberto, Cristian y Alexis, por la separación de las muestras de ambos cruceros. Finalmente, agradecemos los comentarios de los correctores anónimos de este trabajo.

\section{REFERENCIAS}

Anger, K. 2001. The biology of decapod crustacean larvae. Crustacean Issues 14. Ed. Ronald Vonk. A. Balkema, Amsterdam, 419 pp.
Arana, P. 1987. Perspectivas históricas y proyecciones de la actividad pesquera realizada en el archipiélago de Juan Fernández, Chile. En: J.C. Castilla (ed.). Islas oceánicas chilenas: conocimiento científico y necesidades de investigaciones. Ediciones Universidad Católica de Chile, Santiago, pp. 319353.

Atkinson, J. \& N. Boustead. 1982. The complete larval development of the scyllarid lobster Ibacus alticrenatus Bate, 1888 in New Zeland waters. Crustaceana, 42(3): 275-287.

Báez, P. 1973. Larvas phyllosoma del Pacífico sur oriental (Crustacea, Macrura, Scyllaridea). Rev. Biol. Mar., Valparaíso, 15(1): 115-130.

Báez, P. 1979. El puerulus de Jasus frontalis (H. Milne Edwards, 1837) (Crustacea: Decapoda: Palinuridae). Rev. Biol. Mar., Valparaíso, 16(3): 225-228.

Báez, P. 1983. Larvas phyllosoma y puerulus de la langosta verde Panulirus gracilis Streets 1871, procedentes de la expedición Costa Rica, 1973 (Crustacea: Decapoda: Palinuridae). Rev. Biol. Mar., Valparaíso, 19(1): 79-111.

Báez, P. \& R. Ruiz. 2000. Puerulus y postpuerulus de Projasus bahamondei George, 1976 (Crustacea, Decapoda, Palinuridae). Invest. Mar., Valparaíso, 28: $15-25$.

Bahamonde, N. 1987. San Félix y San Ambrosio, las islas llamadas Desventuradas. En: J.C. Castilla (ed.). Islas oceánicas chilenas: conocimiento científico y necesidades de investigaciones. Ediciones Universidad Católica de Chile, Santiago, pp. 85100 .

Castilla, J.C. \& N. Rozbaczylo. 1987. Invertebrados marinos de la isla de Pascua, y Salas y Gómez. En: J.C. Castilla (ed.). Islas oceánicas chilenas: conocimiento científico y necesidades de investigaciones. Ediciones Universidad Católica de Chile, Santiago, pp. 191-215.

Cobb, J. 1997. Oceanic processes affecting lobster larvae: report from a workshop. Mar. Freshw. Res., 48: 771-775.

Coutures, E. 2001. On the first phyllosoma stage of Parribacus caledonicus Holthuis, 1960, Scyllarides squamosus (H. Milne-Edwards, 1837) and Arctides regalis Holthuis, 1963 (Crustacea, Decapoda, Scyllaridae) from New Caledonia. J. Plankton Res., 23(7): 745-751. 
Dupré, E. \& Ch. Guisado.1996. Identificación de los primeros estados de phyllosoma de la langosta de Juan Fernández (Jasus frontalis) mantenidos en laboratorio. Invest. Mar., Valparaíso, 24: 39- 50.

George, R.W. 1997. Tectonic plate movement and the evolution of Jasus and Panulirus spiny lobsters (Palinuridae). Mar. Freshw. Res., 48: 1121-1130.

Gurney, R. 1936. Larvae of decapod Crustacea. Part III. Phyllosoma. Discovery Rep., 12: 400-440.

Ito, M. \& J. Lucas.1990. The complete larval development of the scyllarid lobster, Scyllarus demani Holthuis, 1946 (Decapoda, Scyllaridae), in the laboratory. Crustaceana, 58(2): 144-167.

Johnson, M. 1971. The palinurid and scyllarid lobster larvae of the tropical eastern Pacific and their distribution as related to the prevailing hydrography. Bull. Scripps Inst. Oceanogr., 19: 136.

Kittaka, J., K. Ono \& J. Booth. 1997. Complete development of the green rock lobster, Jasus verreauxi from egg to juvenile. Bull. Mar. Sci., 61(1): 57-71.

Mann, K. \& J. Lazier. 1991. Dynamics of marine ecosystems. Biological-physical interactions in the oceans. Blackwell Scientific Publications, Boston, 466 pp.

McWilliam, P. 1995. Evolution in the phyllosoma and puerulus phases of the spiny lobster genus Panulirus White. J. Crust. Biol., 15(3): 542-557.

Menzies, R. \& J. Kerrigan. 1978. Implications of spiny lobster recruitment patterns of the CaribbeanA biochemical genetic approach. Proceedings of the Gulf and Caribbean Fisheries Institute, 31: 164178.

Moraga, J. \& J. Olivares. 1996. Hidrografía del área costera de isla de Pascua, mayo 1994. Cienc. Tecnol. Mar, 19: 47-56.

Mujica, A. 1993. Zooplancton de las aguas circundantes a la isla de Pascua (270' S-109²6'W). Cienc. Tecnol. Mar, 16: 55-61.

Ohman, M. 1988. Behavioral responses of zooplankton to predation. Bull. Mar. Sci., 43(3): 530-550.

Palma, S. 1994. Distribución y abundancia de larvas de langostino colorado Pleuroncodes monodon frente a la costa de Concepción, Chile. Invest. Mar., Valparaíso, 22: 13-29.

Palma, S., J. Meruane \& A. Mujica. 1976. Observaciones sobre el meroplancton del archipiélago de Juan Fernández. Enero 1974. Cienc. Tecnol. Mar, 2: 117-126.

Parin, N., A. Mironov \& K. Nesis. 1997. Biology of Nazca and Sala y Gómez submarine ridges, an outpost of the Indo-West Pacific fauna in the Eastern Pacific ocean: composition and distribution of the fauna, its communities and history. Adv. Mar. Biol., 32: 147-230.

Parson, T., M. Takahashi \& B. Hargrave. 1984. Biological oceanographic processes. Pergamon Press, New York, 330 pp.

Phillip, B., D. Rimmer \& D. Reid. 1978. Ecological investigations of the late stage phyllosoma and puerulus larvae of the western rock lobster Panulirus longipes cygnus. Mar. Biol., 45: 347-357.

Pollock, D. 1990. Paleocenography and speciation in the spiny lobster genus Jasus. Bull. Mar. Sci., 46: 387-405.

Pollock, D. 1992. Paleocenography and speciation in the spiny lobster genus Panulirus in the IndoPacific. Bull. Mar. Sci., 51(2): 135-146.

Pollock, D. 1995. Evolution of life-history patterns in three genera of spiny lobster. Bull. Mar. Sci., 57(2): 516-526.

Retamal, M. 1981. Catálogo ilustrado de los crustáceos decápodos de Chile. Gayana, Zool., 44: 1-110.

Retamal, M. 1994. Los decápodos de Chile. Universidad de Concepción, Depto. de Oceanografía, Concepción, 256 pp.

Retamal, M. 2000a. (CD-ROM) Decápodos de Chile. ETI-Universidad de Concepción. SpringerVerlag, Berlín.

Retamal, M. 2000b. Arctides regalis Holthuis, 1963 (Scyllaridae, Arctidinae) una nueva "langosta chata” en aguas oceánicas chilenas. Bol. Soc. Biol. Concepción, Chile, 71: 45-47.

Richards, W. \& J. Goulet. 1976. An operational surface drift model used for studying larval lobster recruitment and dispersal. FAO Fish. Rep., 200: 363-374. 
Rimmer, D. \& B. Phillips. 1979. Diurnal migration and vertical distribution of phyllosoma larvae of the western rock lobster Panulirus cygnus. Mar. Biol., 54: 109-124.

Ritz, D. \& L. Thomas. 1973. The larval and post-larval stages of Ibacus peronii Leach (Decapoda, Reptantia, Scyllaridae). Crustaceana, 24(1): 5-16.

Rozbaczylo, N. \& J.C. Castilla. 1987. Invertebrados marinos del archipiélago de Juan Fernández. En: J.C. Castilla (ed.). Islas oceánicas chilenas: conocimiento científico y necesidades de investigaciones. Ediciones Universidad Católica de Chile, Santiago, pp 167-189.

Saisho, T. 1966. Studies on the phyllosoma larvae with reference to the oceanographic conditions. Mem. Fac. Fish. Kagoshima Univ., 15: 177-239.

Sastry, A. 1982. Pelagic larval ecology and development. En: D.E. Bliss (ed.). The biology of Crustacea. Academic Press, New York, 2: 213-282.

Sievers, H. \& N. Silva. 1975. Masas de agua y circulación en el océano Pacífico Sud Oriental, latitudes $18^{\circ} \mathrm{S}-33^{\circ} \mathrm{S}$. (Operación oceanográfica "Marchile VIII"). Cienc. Tecnol. Mar, 1: 7-67.

Silva, N. 1992. Condiciones oceanográficas alrededor de la Isla de Pascua durante la primavera de 1979. Cienc. Tecnol. Mar, 15: 21-30.

Silva, N. \& D. Konow. 1975. Contribución al conocimiento de las masas de agua en el Pacífico Sudoriental, Expedición Krill. Crucero 3-4. julioagosto 1974. Rev. Com. Perm. Pacífico Sur, 3: 6375.

Recibido: 12 abril 2004; Aceptado: 10 septiembre 2004
Silva, N. \& H. Sievers. 1974. Masas de agua, velocidad geostrófica y transporte de volumen entre Valparaíso e Isla Robinson Crusoe (Océano Pacífico Sudoriental). Rev. Com. Perm. Pacífico Sur, 2: 102120.

Vereschaka, A.L. 1990. Pelagic decapods from semount of Nazca and Sala-I-Gomez ridges. En: A.N. Mironov \& J.A. Rudjacov (eds.). Plankton and benthos from the Nazca and Sala-y-Gomez submarine ridges. Trud. Inst. Okeanol, Acad. Sci., Moscow, 124: 129-155.

Vereschaka, A.L. 1995. Macroplankton in the nearbottom layer of continental slopes and seamounts. Deep-Sea Res., 42(9): 1639-1668.

Webber, W. \& J. Booth. 1988. Projasus parkeri (Stebbing, 1902) (Crustacea, Decapoda, Palinuridae) in New Zeland and description of a Projasus puerulus from Australia. Nat. Mus. N.Z. Records, 3(8): 81-92.

Wehrtmann, I. \& P. Báez. 1997. Larvas y estadios tempranos de desarrollo de crustáceos decápodos de Chile. Descripciones publicadas. Invest. Mar., Valparaíso, 25: 263-276.

Yeung, C. \& M. McGowan. 1991. Differences in inshore-offshore and vertical distribution of phyllosoma larvae of Panulirus, Scyllarus and Scyllarides in Florida keys in May-June, 1989. Bull. Mar. Sci., 49(3): 699-714. 